\title{
ON THE UNCERTAINTY INEQUALITY AS APPLIED TO DISCRETE SIGNALS
}

\author{
Y. V. VENKATESH, S. KUMAR RAJA, AND G. VIDYASAGAR \\ Received 11 June 2005; Revised 24 October 2005; Accepted 28 November 2005
}

Given a continuous-time bandlimited signal, the Shannon sampling theorem provides an interpolation scheme for exactly reconstructing it from its discrete samples. We analyze the relationship between concentration (or compactness) in the temporal/spectral domains of the (i) continuous-time and (ii) discrete-time signals. The former is governed by the Heisenberg uncertainty inequality which prescribes a lower bound on the product of effective temporal and spectral spreads of the signal. On the other hand, the discrete-time counterpart seems to exhibit some strange properties, and this provides motivation for the present paper. We consider the following problem: for a bandlimited signal, can the uncertainty inequality be expressed in terms of the samples, using the standard definitions of the temporal and spectral spreads of the signal? In contrast with the results of the literature, we present a new approach to solve this problem. We also present a comparison of the results obtained using the proposed definitions with those available in the literature.

Copyright @ 2006 Hindawi Publishing Corporation. All rights reserved.

\section{Introduction}

We deal with real functions (signals) $f$ of the real variable $x \in \mathbb{R}:\left\{f(x): f(x) \in \mathbb{L}_{2}\right\}$, that is, the class of square integrable functions, having unit energy and centered at the origin. The independent variable $x$ can denote either time (for dealing with time-dependent phenomena) or space (for describing space-dependent functions like images). In what follows, we use the terms "space" and "time" interchangeably.

With $F(\omega)$ denoting the Fourier transform of $f(x)$, we have, based on the assumptions made above, the following identities:

$$
\int_{-\infty}^{\infty}|f(x)|^{2} \mathrm{~d} x=1=\frac{1}{2 \pi} \int_{-\infty}^{\infty}|F(\omega)|^{2} \mathrm{~d} \omega .
$$

The space localization of a signal is described by its "effective spatial width" $\left(\Delta_{x}\right)$ :

$$
\left(\Delta_{x}\right)^{2}=\frac{\int_{-\infty}^{\infty} x^{2} f^{2}(x) \mathrm{d} x}{\int_{-\infty}^{\infty} f^{2}(x) \mathrm{d} x}=\int_{-\infty}^{\infty} x^{2} f^{2}(x) \mathrm{d} x .
$$

Hindawi Publishing Corporation International Journal of Mathematics and Mathematical Sciences Volume 2006, Article ID 48185, Pages 1-22

DOI 10.1155/IJMMS/2006/48185 
2 On the uncertainty inequality as applied to discrete signals

Similarly, the frequency localization of a signal is described by its "effective spectral width" in terms of $F(\omega)$ as follows:

$$
\left(\Delta_{\omega}\right)^{2}=\frac{\int_{-\infty}^{\infty} \omega^{2}|F(\omega)|^{2} \mathrm{~d} \omega}{\int_{-\infty}^{\infty}|F(\omega)|^{2} \mathrm{~d} \omega}=\frac{1}{2 \pi} \int_{-\infty}^{\infty} \omega^{2}|F(\omega)|^{2} \mathrm{~d} \omega .
$$

One of the fundamental properties of continuous signals is that $f(x)$ and $F(\omega)$ cannot both be of short duration. This is made explicit (i) qualitatively by the scaling theorem,

$$
a f(a x) \Longleftrightarrow F\left(\frac{\omega}{a}\right)
$$

and (ii) quantitatively by the uncertainty principle which places a lower bound on the product of effective spatial and spectral widths of continuous signals [15]:

$$
\left(\Delta_{x}\right)^{2}\left(\Delta_{\omega}\right)^{2} \geq \frac{1}{4}
$$

The equality in (1.5) is satisfied only by the Gaussian function. For a survey on the uncertainty inequality, see [12]; and for related results as applied to wavelet transforms, and time-frequency representations, see [3-5].

The motivation for the work presented in this paper arose from the results obtained in $[1,14]$ by applying the above inequality (with modifications to the definitions of effective spreads in the time and frequency domains) to discrete signals.

In the discrete case, the effective spatial width $D_{n}$ and spectral width $D_{\omega}$ are defined (along the lines of the counterparts of the continuous case) by $[1,14]$ as follows:

$$
D_{n}^{2}=\sum_{n=-\infty}^{\infty} n^{2}|f[n]|^{2} ; \quad D_{\omega}^{2}=\int_{-\pi}^{\pi} \omega^{2}\left|F\left(e^{j \omega}\right)\right|^{2} d \omega .
$$

We find that there are discrete signals for which there is no strict positive lower bound for the space-bandwidth product. For instance, the sequence

$$
\delta(n)= \begin{cases}0, & \forall n \neq 0 \\ 1, & n=0\end{cases}
$$

has zero space-bandwidth product. (Recall that there is no continuous version of the signal with such a property.)

However, the authors of $[1,14]$ propose some additional restrictions on discrete signals in order to arrive at satisfactory definitions of effective widths in the spatial (i.e., original) and spectral domains. On the other hand, DeBrunner et al. $[6,16]$ propose an entropy-based measure for the simultaneous localization of finite-duration discretetime signals in the time-frequency plane. Also, for specially defined second moments, Doroslovački et al. $[10,11]$ show that the Gabor uncertainty relation is satisfied for any finite-energy signal. To arrive at the result, the authors use the "convolution-invariance" condition. 
In contrast, Donoho and Huo [8] describe an uncertainty principle that deals with sparse representation of discrete-time signals in multiple bases. However, their result does not refer to uncertainty in the sense we are using the term here (i.e., time-frequency localization).

Even though the sparsity inequality of [9] and other information-theoretic inequalities can be related to uncertainty (see [7]), it is found that the following problems deserve to be considered in their own right.

(1) Suppose we are given the appropriately sampled values of a bandlimited signal. Can we express the space-bandwidth product (SBP) (1.5) in terms of these samples, using the standard definitions of the spatial and spectral spreads (meant for the continuous version) of the signal, unlike the results of $[1,14]$ ? A solution to this problem implies consistency in the applications of the definitions (of spatial and spectral widths) to continuous and discrete versions of the signal. By the word "consistent," we mean the following. Suppose we define, in a certain fashion, the SBP of a continuous, bandlimited signal; and compute the SBP with reference to this signal. We then sample the signal, and, for the discrete signal, compute the discrete SBP, defined in a manner analogous to that of the continuous signal. The two definitions are consistent if the results of the two computations are the same (except, possibly, for numerical errors).

(2) Does there exist an optimal bandlimited signal for which (1.5) is an equality? If the answer is affirmative, what is it?

In contrast with the results of the literature, we present a new approach to solve the first problem. A mathematical treatment of the second problem still seems to be open. However, we provide some experimental results that point towards a "filtered Gaussian" as the solution to the second problem. For more details, see Section 5.

The outline of the paper is as follows. Section 2 briefly surveys the results of Ishii and Furukawa [14], Calvez and Vible [1], DeBrunner et al. [6, 16], and Doroslovački et al. $[10,11]$. Section 3 contains our approach to compute spatial and spectral widths from discrete samples, followed by Section 4 which compares the new results with those of Doroslovački et al. $[10,11]$. Section 5 presents some experimental findings related to the discovery of an optimal bandlimited signal. Finally, Section 6 concludes the paper.

\section{Brief survey of existing results}

As indicated above, some authors extend the uncertainty inequality to discrete signals by carrying over the definitions of the space- and the frequency-domain spreads, from the continuous to the discrete case $[1,14]$, while others employ "specially defined second moments" $[10,11]$. Ishii and Furukawa [14] define the space and spectral spreads for a discrete-space signal using (1.6). Based on these definitions and the assumptions given below, they obtain an uncertainty inequality. Here is a brief summary of these results from the literature.

Assumptions. (i) $f(x)$ is a bandlimited analog signal, that is, $F(\omega)=0$, for all $|\omega| \geq \sigma$.

(ii) $f[n]$ is obtained by sampling $f(x)$ at Nyquist rate, that is, $f[n]=f(n X)$, where $X=\pi / \sigma$. 
4 On the uncertainty inequality as applied to discrete signals

(iii) Let $F_{d}\left(e^{j \Omega}\right)$ denote the Fourier transform of $f[n]$ :

$$
F_{d}\left(e^{j \Omega}\right)=\sum_{n=-\infty}^{\infty} f[n] e^{-j \Omega n}
$$

from which

$$
f[n]=\frac{1}{2 \pi} \int_{-\pi}^{\pi} F_{d}\left(e^{j \Omega}\right) e^{j \Omega n} \mathrm{~d} \Omega .
$$

(iv) Total energy of the signal is one, that is,

$$
\sum_{n=-\infty}^{\infty}|f[n]|^{2}=\frac{1}{2 \pi} \int_{-\pi}^{\pi}\left|F_{d}\left(e^{j \Omega}\right)\right|^{2} \mathrm{~d} \Omega=1 .
$$

(v) $\Omega=\omega X$.

For a proof of the following uncertainty inequality, see [14].

$$
D_{n} D_{\Omega} \geq \frac{1}{\sqrt{2 \pi}}\left|\pi F_{d}^{2}\left(e^{j \pi}\right)-\frac{1}{2} \int_{-\pi}^{\pi} F_{d}^{2}\left(e^{j \Omega}\right) \mathrm{d} \Omega\right| .
$$

Further, under the following assumptions,

(a) $F(\omega)=0, \omega= \pm \sigma$;

(b) $F_{d}\left(e^{j \Omega}\right)$ is real,

the uncertainty inequality (2.4) simplifies to

$$
D_{n} D_{\Omega}>\sqrt{\frac{\pi}{2}}
$$

Though the inequality (2.5) appears to be a satisfactory result, it holds only under the assumptions (a) and (b) mentioned above. On the other hand, though (2.4) holds for all finite-energy signals, the lower bound could be zero. For example, for the unit sample sequence (1.7), which is obtained by sampling the continuous function

$$
\frac{\sin (\sigma \pi x)}{\sigma \pi x}
$$

at Nyquist rate, $D_{n}$ is zero. Therefore, in effect, the lower bound of the uncertainty product of any finite-energy signal is 0 . In other words,

$$
D_{n} D_{\Omega} \geq 0
$$

Further, the continuous version (2.6) has $\infty$ as the uncertainty product, whereas the corresponding value for the discrete version $\delta[n]$ is 0 ! In contrast, DeBrunner et al. [6] define an entropy-based uncertainty measure (see below) in their attempt to describe timefrequency localization of discrete-time signals. Further, they conjecture an uncertainty inequality which they refine to a theorem in [17]. 
Definitions. (1) Let $H_{N}$ denote the Hilbert space of $N$-length sequences $\{u \mid u:\{0,1,2, \ldots$, $N-1\} \rightarrow \mathbb{C}\}$ with the norm defined by $\|u\|^{2}=\sum_{n=0}^{N-1}|u(n)|^{2}$.

(2) For $u \in H_{N},\|u\|=1$, define

$$
H(u)=-\sum_{n=0}^{N-1}|u(n)|^{2} \ln \left(|u(n)|^{2}\right) ;
$$

$H(u)$ is the measure of (lack of) concentration of $u$ in the domain $n$.

(3) Let $F(u)=\widehat{u}(k)=(1 / \sqrt{N}) \sum_{n=0}^{N-1} u(n) W_{N}^{k n}$, where $W_{N}=e^{-(j 2 \pi / N)}$, denote the DFT of $u$.

Define

$$
H(F(u))=H(\widehat{u}(k))=-\sum_{k=0}^{N-1}|\widehat{u}(k)|^{2} \ln \left(|\widehat{u}(k)|^{2}\right)
$$

$H(F(u))$ is the measure of (lack of) concentration of $F(u)$ in the frequency domain.

(4) Define

$$
H_{p}(u)=p H(u)+(1-p) H(F(u)), \quad\left(u \in H_{N},\|u\|=1,0 \leq p \leq 1\right) ;
$$

$H_{p}(u)$ is the measure of concentration of the signal in the $n-k$ plane.

Using the above concentration measures, Przebinda et al. [17] show that the following relation holds:

$$
H_{1 / 2}(u) \geq \frac{1}{2} \ln (N)
$$

which is the Hirschman uncertainty principle [7].

However, the entropy-based definitions of spatial and spectral spreads found in [6] are different from those used in this paper. In $[6,16]$, the authors argue in favor of entropy-based concentration measures for discrete signals, and these measures may refer to localization in discrete-time and discrete-frequency spaces. We must emphasize that the discrete Hirschman uncertainty principle places a lower bound on the sum of the concentration measures, and not on the product. In contrast, we deal with the product of (variance-based) concentration measures (in the sense used in the Gabor uncertainty principle) which seems to be quite distinct from the summation of concentrations. Moreover, it is also not clear how the latter is related to the product of variance-based concentration measures.

In contrast to DeBrunner, Doroslovački [10] attempts to give a unified framework for defining the spatial and spectral spreads for the continuous- and discrete-space signals, in the sense that a generalized uncertainty inequality is derived for both.

THEOREM 2.1. Let $B(\omega)$ be a complex-valued function, and $A(\omega)$ a real-valued function on $[-p, p]$ such that $B(\omega) F(\omega)$ is absolutely continuous,

$$
\begin{gathered}
A(\omega)=\int_{\omega_{0}}^{\omega} \frac{1}{|B(\nu)|^{2}} \mathrm{~d} \nu, \\
A(\omega)|B(\omega) F(\omega)|^{2}=0 \quad \text { for } \omega= \pm p .
\end{gathered}
$$


6 On the uncertainty inequality as applied to discrete signals

Also, define

$$
\begin{aligned}
& m_{2}=\frac{1}{2 \pi E_{f}} \int_{-p}^{p}\left|[B(\omega) F(\omega)]^{\prime}\right|^{2} \mathrm{~d} \omega \\
& M_{2}=\frac{1}{2 \pi E_{f}} \int_{-p}^{p}|A(\omega) B(\omega) F(\omega)|^{2} \mathrm{~d} \omega .
\end{aligned}
$$

Then,

$$
m_{2} M_{2} \geq \frac{1}{4}
$$

Moreover, the inequality becomes equality for

$$
F(\omega)=\frac{F\left(\omega_{1}\right) B\left(\omega_{1}\right)}{B(\omega)} \exp \left(K \int_{\omega_{1}}^{\omega} A(\nu) \mathrm{d} \nu\right)
$$

where $K \in \mathbb{R}$, and it is constrained by the finite-energy requirement. See [10] for its proof.

Note that (2.14) is the generalized second moment of the signal in the time domain, and (2.15) is the generalized second moment in the frequency domain; and (2.16) is the generalized uncertainty inequality. For different choices of $p$, the theorem is valid for both the continuous-time and the discrete-time signals. In other words, for continuous signals $p=\infty$, and for discrete signals $p=\pi$. Also, the Gabor uncertainty relation for the point $(0,0)$ in the time-frequency plane is obtained by setting $B(\omega)=1$ and $\omega_{0}=0$. However, condition (2.13) and the uncertainty relation (2.16) are not satisfied, in general, in the discrete-time case, except when $|F( \pm \pi)|=0$. This particular result is similar to the one obtained in $[1,14]$.

Even though there are many choices for $B(\omega)$ satisfying the given conditions, any specific $B(\omega)$ is restricted by the requirement that the product of spectra of two optimal functions must be the spectrum of another optimal function. Since, in the spatial domain, the product corresponds to the convolution of optimal functions, the set of optimal functions will be invariant under convolution. This requirement on $B(\omega)$ is termed as the convolution-invariance condition. In order to satisfy this condition, the following equation is to be solved:

$$
B(\omega)=G^{K}(\omega)
$$

where $G(\omega)$ is the optimal function. Under this condition, Doroslovački [10] proves the following.

Proposition 2.2. The relations (2.16) and (2.18) are jointly satisfied for all finite-energy absolutely continuous functions $F(\omega)$, only (a) in the continuous-time case $B(\omega)=1$, and (b) in the discrete-time case $B(\omega)=\cos (\omega / 2)$.

Using the above proposition, the following uncertainty relations are obtained for the continuous-time and discrete-time signals. 
Table 2.1. Comparison of spatial and spectral widths.

\begin{tabular}{cc|c|cc|c|cc}
\hline & & Continuous & \multicolumn{2}{|c|}{ Discrete } & Continuous & \multicolumn{2}{c}{ Discrete } \\
\hline & $\begin{array}{c}\text { Gabor's } \\
\text { Def. }\end{array}$ & $\begin{array}{c}\text { Doroslovački's } \\
\text { Def. }\end{array}$ & $\begin{array}{c}\text { Proposed } \\
\text { Def. }\end{array}$ & $\begin{array}{c}\text { Gabor's } \\
\text { Def. }\end{array}$ & $\begin{array}{c}\text { Doroslovački's } \\
\text { Def. }\end{array}$ & $\begin{array}{c}\text { Proposed } \\
\text { Def. }\end{array}$ \\
\hline \multirow{2}{*}{ no. } & Function & $\begin{array}{c}\text { Spatial } \\
\text { width }\end{array}$ & $\begin{array}{c}\text { Spatial } \\
\text { width }\end{array}$ & $\begin{array}{c}\text { Spatial } \\
\text { width }\end{array}$ & $\begin{array}{c}\text { Spectral } \\
\text { width }\end{array}$ & $\begin{array}{c}\text { Spectral } \\
\text { width }\end{array}$ & $\begin{array}{c}\text { Spectral } \\
\text { width }\end{array}$ \\
\hline 1 & sinc & $\infty$ & Not applicable & $\infty$ & 0.33333 & Not applicable & 0.33333 \\
2 & Triangle & 0.74288 & 0.81777 & 0.76015 & 0.40020 & 0.07950 & 0.39831 \\
3 & Halfcos & 2.46585 & 0.07961 & 2.47144 & 0.13076 & 0.05068 & 0.13069 \\
\hline
\end{tabular}

Uncertainty inequality — continuous version:

$$
\left\{\frac{1}{E_{f}} \int_{-\infty}^{\infty} x^{2}|f(x)|^{2} \mathrm{~d} x\right\}\left\{\frac{1}{2 \pi E_{f}} \int_{-\infty}^{\infty} \omega^{2}|F(\omega)|^{2} \mathrm{~d} \omega\right\} \geq \frac{1}{4}
$$

Uncertainty inequality - discrete version:

$$
\left\{\frac{1}{2 \pi E_{f}} \int_{-\pi}^{\pi}\left|\left[\cos \left(\frac{\omega}{2}\right) F(\omega)\right]^{\prime}\right|^{2} \mathrm{~d} \omega\right\}\left\{\frac{1}{2 \pi E_{f}} \int_{-\pi}^{\pi} 4 \sin ^{2}\left(\frac{\omega}{2}\right)|F(\omega)|^{2} \mathrm{~d} \omega\right\} \geq \frac{1}{4} .
$$

See [10] for the proofs.

Remarks. (i) The choice of $B(\omega)$ based on the convolution-invariance condition does not seem to be clear.

(ii) It is also not clear why the convolution-invariance property of the optimal functions is chosen while the optimal function in the continuous-space case (Gaussian) has other properties as well.

(iii) In Table 2.1, numerical values of effective widths computed for various bandlimited functions, using the definitions given in (2.14) and (2.15), have been given. It is found that the uncertainty inequalities for the continuous- and discrete-space cases are inconsistent in the sense described in the last sentence of Section 1, item (1).

(iv) The restriction on the spectra of the continuous and discrete functions is that they should be absolutely continuous. However, the $\operatorname{sinc}$-function $(\sin (\pi x) / \pi x)$ has a discontinuous spectrum, while the discrete $\delta[n]$ has a continuous spectrum. Therefore, in this case, it appears that Doroslovački's definitions cannot be applied.

To summarize some of the relevant results of the literature, the uncertainty inequality, as found in the continuous domain, can be extended to the discrete domain only after modifying the definitions of spread (in the time and frequency domains). Motivated by such a limitation of some of the results of the literature, we present, in the next section, a new approach, for bandlimited signals, in which we modify the interpolating function used in the sampling theorem, and reformulate the standard uncertainty inequality in terms of the samples. 


\section{Proposed approach}

Let $\{f[n]\}_{n \in \mathbb{Z}}$ denote the samples obtained by sampling uniformly a bandlimited signal $f(x)$ with the sampling interval $X$. Also, let the Fourier transform $F(\omega)$ of the signal satisfy the condition

$$
F(\omega)=0, \quad \forall|\omega| \geq \sigma
$$

Assuming that the sampling interval $X$ satisfies the condition $X<\pi / \sigma$, the original signal $f(x)$ can be reconstructed from its samples using the standard interpolation formula

$$
f(x)=\sum_{n \in \mathbb{Z}} f(n X) g(x-n X), \quad g(x)=\frac{\sin ((\pi / X) x)}{(\pi / X) x} .
$$

When we try to express the Heisenberg inequality directly in terms of the samples of a bandlimited signal, it is found that the standard interpolating sinc-function is inappropriate even though it does the job of interpolation perfectly. The difficulty in invoking the same function to achieve our goal (of computing the space-bandwidth product directly from the samples) arises from the nondifferentiability property of the spectrum (which is the ideal lowpass filter) of the sinc-function. Note that differentiability of the spectrum of the sinc-function is needed in expressing the effective spatial width of the signal. See (3.10) for the expression related to the effective spatial width of the signal in terms of its samples. The evaluation of the integral on the right-hand side of (3.10) leads to computation of the derivative of the spectrum of the sinc-function.

The main contribution of the paper is the refinement of the sinc-function in order to compute the lower bound on the uncertainty of a bandlimited signal in terms of its discrete samples. It should be noted here that we have not altered the sampling definition. We have only modified the interpolating function, without affecting the reconstruction of the original signal. In what follows, we first compute the effective spectral width of the signal in terms of its samples, and then consider the problem of expressing its effective spatial width by refining the sinc-function.

3.1. Refinement of the sinc-function. Without any loss of generality, we assume that the signal has unit energy and is centered. The effective widths of the signal (continuous) in the spatial and frequency domains are defined by (1.2) and (1.3). Since the signal is bandlimited $(\sigma<\pi / X)$, the lower and upper limits of the integrals in (1.3) can be replaced by $-\pi / X$ and $\pi / X$, respectively.

The discrete-space Fourier transform $F_{d}(\Omega)$ for the discrete samples $\{f[n]\}_{n \in \mathbb{Z}}$ is defined by (2.1). It can be shown that $F_{d}(\Omega)$ and $F(\omega)$ are related by

$$
F_{d}(\Omega)=\frac{1}{X} F(\omega), \quad \forall|\omega| \leq \frac{\pi}{X} .
$$

Using the above relation, and substituting $\Omega=\omega X$ in (1.3), we get

$$
\begin{aligned}
\left(\Delta_{\omega}\right)^{2} & =\frac{1}{2 \pi} \int_{-\pi}^{\pi}\left(\frac{\Omega}{X}\right)^{2} X^{2}\left|F_{d}(\Omega)\right|^{2} \frac{\mathrm{d} \Omega}{X} \\
& =\frac{1}{2 \pi X} \int_{-\pi}^{\pi} \Omega^{2}\left|F_{d}(\Omega)\right|^{2} \mathrm{~d} \Omega .
\end{aligned}
$$


Using (2.1), we can express $\left|F_{d}(\Omega)\right|^{2}$ in terms of the samples as

$$
\begin{aligned}
\left|F_{d}(\Omega)\right|^{2} & =\left(\sum_{m \in \mathbb{Z}} f[m] \exp (-j \Omega m)\right)\left(\sum_{n \in \mathbb{Z}} f[n] \exp (-j \Omega n)\right)^{*} \\
& =\sum_{m \in \mathbb{Z}} \sum_{n \in \mathbb{Z}} f[m] f[n] \exp (j \Omega(m-n)),
\end{aligned}
$$

where the superscript $*$ denotes complex conjugate. Use (3.5) in (3.4) to get

$$
\left(\Delta_{\omega}\right)^{2}=\frac{1}{2 \pi X} \int_{-\pi}^{\pi} \Omega^{2} \sum_{m \in \mathbb{Z}} \sum_{n \in \mathbb{Z}} f[m] f[n] \exp (j \Omega(m-n)) \mathrm{d} \Omega .
$$

Assuming that the double summation inside the integral of (3.6) converges uniformly for all $\Omega$, we rewrite (3.6) as

$$
\left(\Delta_{\omega}\right)^{2}=\frac{1}{2 \pi X} \sum_{m \in \mathbb{Z}} \sum_{n \in \mathbb{Z}} f[m] f[n] \int_{-\pi}^{\pi} \Omega^{2} \exp (j \Omega(m-n)) \mathrm{d} \Omega .
$$

It can be shown that the above expression for the effective spectral width of $f(x)$ can be expressed in terms of its samples:

$$
\left(\Delta_{\omega}\right)^{2}=\int_{-\pi}^{\pi}|\omega F(\omega)|^{2} \mathrm{~d} \omega=\frac{1}{2 \pi X}\left[\frac{2 \pi^{3}}{3} \sum_{n \in \mathbb{Z}} f^{2}[n]+\sum_{\substack { m \in \mathbb{Z} \\
\begin{subarray}{c}{n \in \mathbb{Z} \\
m \neq n{ m \in \mathbb { Z } \\
\begin{subarray} { c } { n \in \mathbb { Z } \\
m \neq n } }\end{subarray}} \frac{4 \pi(-1)^{(m-n)}}{(m-n)^{2}} f[m] f[n]\right] .
$$

The effective spatial width of the signal is defined in (1.2) from which, by using (3.2) in the general form (i.e., for a general interpolating function, $g(x)$ ), we get

$$
\begin{aligned}
\left(\Delta_{x}\right)^{2} & =\int_{-\infty}^{\infty}\left(x \sum_{m \in \mathbb{Z}} f(m X) g(x-m X)\right)\left(x \sum_{n \in \mathbb{Z}} f(n X) g(x-n X)\right) \mathrm{d} x \\
& =\int_{-\infty}^{\infty} \sum_{m \in \mathbb{Z}} \sum_{n \in \mathbb{Z}} x f(m X) g(x-m X) x f(n X) g(x-n X) \mathrm{d} x .
\end{aligned}
$$

Interchanging the order of summation and integration (assuming uniform convergence of the summation) in the above equation, we get

$$
\begin{aligned}
\left(\Delta_{x}\right)^{2} & =\sum_{m \in \mathbb{Z}} \sum_{n \in \mathbb{Z}} \int_{-\infty}^{\infty} f(m X) x g(x-m X) f(n X) x g(x-n X) \mathrm{d} x \\
& =\sum_{m \in \mathbb{Z}} \sum_{n \in \mathbb{Z}} f(m X) f(n X) \int_{-\infty}^{\infty} x g(x-m X) x g(x-n X) \mathrm{d} x .
\end{aligned}
$$


The integral (on the right-hand side of (3.10)) inside the summation can be evaluated as follows. Let $G(\omega)$ denote the Fourier transform of $g(x)$. The Fourier transform of $(g-$ $n X)$ is given by $G(\omega) \exp (-j \omega n X)$. If we employ, as it is done in the standard literature, the sinc-function for $g(x)$, the integral of (3.10) cannot be evaluated. Therefore, we impose the following restrictions on $G(\omega)$.

(1) It must be real and differentiable in the interval $(-\sigma-\epsilon, \sigma+\epsilon)$, where $\epsilon$ is a positive number (as small as possible) such that $\epsilon<\pi / X-\sigma$. Note that this condition on $\epsilon$ is mandatory for perfect reconstruction.

(2) $G(\sigma)=X, G(\sigma+\epsilon)=0, G(-\sigma)=X, G(-\sigma-\epsilon)=0$.

(3) $\mathrm{d} G(\omega) /\left.\mathrm{d} \omega\right|_{\sigma}=0, \mathrm{~d} G(\omega) /\left.\mathrm{d} \omega\right|_{-\sigma}=0$.

(4) $G(\omega)=X$, a constant in the interval $(-\sigma, \sigma)$.

We call the above four restrictions "smoothness conditions" on $G$. There are many ways of selecting $G(\omega)$, one of which is the following function:

$$
G(\omega)= \begin{cases}\frac{X}{2}\left[\cos \left(\frac{\pi(\omega+\sigma)}{\epsilon}\right)+1\right], & -\sigma-\epsilon \leq \omega<-\sigma, \\ X, & -\sigma \leq \omega \leq \sigma, \\ \frac{X}{2}\left[\cos \left(\frac{\pi(\omega-\sigma)}{\epsilon}\right)+1\right], & \sigma<\omega \leq \sigma+\epsilon, \\ 0, & \text { elsewhere. }\end{cases}
$$

See Figure 3.1 for a sketch of $G(\omega)$. With respect to this choice of the interpolating function, we note the following.

(i) The signal is not modified by redefining the domain of the interpolating function. The extension of the domain (of the interpolating function) has been done in order to compute the integrals involving squares of $x g(x-n X)$ as the integrand.

(ii) The modified interpolation function performs not only perfect reconstruction but also facilitates computation of effective space width. Therefore, we compute the effective space width of the original signal itself, and not of a modified signal.

(iii) The tapered cosine (Tukey) window [13], which is used in the design of FIR filters, also has a profile similar to $G(\omega)$ above. However, it is to be noted that we use $G(\omega)$ for an entirely different purpose-that of reconstructing the bandlimited signal from its samples.

And the other possible $G$ function is as follows:

$$
G(\omega)= \begin{cases}X\left(1-\frac{2}{\epsilon^{2}}(\omega+\sigma)^{3}-\frac{3}{\epsilon^{2}}(\omega+\sigma)^{2}\right), & -\sigma-\epsilon \leq \omega<-\sigma, \\ X, & -\sigma \leq \omega \leq \sigma, \\ X\left(1+\frac{2}{\epsilon^{3}}(\omega-\sigma)^{3}-\frac{3}{\epsilon^{2}}(\omega-\sigma)^{2}\right), & \sigma<\omega \leq \sigma+\epsilon, \\ 0, & \text { elsewhere. }\end{cases}
$$




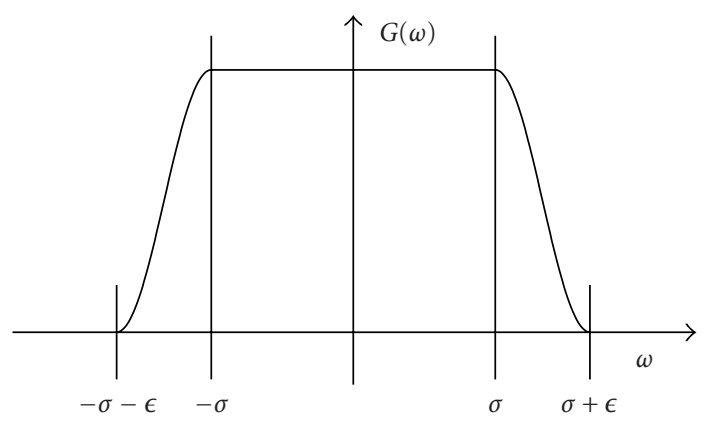

Figure 3.1. A (rough) sketch of $G(\omega)$.

Since $G(\omega)$ is now differentiable, the Fourier transform $H_{1}(\omega)$ of $x g(x-n X)$ is given by

$$
\begin{aligned}
H_{1}(\omega) & =j \frac{\mathrm{d}}{\mathrm{d} \omega}[G(\omega) \exp (-j \omega n X)] \\
& =j \frac{\mathrm{d} G(\omega)}{\mathrm{d} \omega} \exp (-j \omega n X)+n X G(\omega) \exp (-j \omega n X) .
\end{aligned}
$$

Similarly, $H_{2}(\omega)$, the Fourier transform of $x g(x-m X)$, is given by

$$
H_{2}(\omega)=j \frac{\mathrm{d} G(\omega)}{\mathrm{d} \omega} \exp (-j \omega m X)+m X G(\omega) \exp (-j \omega m X) .
$$

Using (3.13) and (3.14), the integral (3.10) can be rewritten as

$$
\int_{-\infty}^{\infty} x g(x-m X) x g(x-n X) \mathrm{d} x=\frac{1}{2 \pi} \int_{-\infty}^{\infty} H_{1}(\omega) H_{2}^{*}(\omega) \mathrm{d} \omega .
$$

The integrand on the right-hand side of (3.15) can be written as

$$
\begin{aligned}
& H_{1}(\omega) H_{2}^{*}(\omega) \\
& =\left(j(m-n) X G(\omega) \frac{\mathrm{d} G(\omega)}{\mathrm{d} \omega}+(m X)(n X)|G(\omega)|^{2}+\left|\frac{\mathrm{d} G(\omega)}{\mathrm{d} \omega}\right|^{2}\right) \exp (j \omega(m-n) X) \\
& \begin{array}{llr}
\Downarrow & \Downarrow & \Downarrow \\
I_{1} & I_{2} & I_{3} .
\end{array}
\end{aligned}
$$

Let the integrals involving the first, second, and third terms above be denoted by $I_{1}, I_{2}, I_{3}$, respectively. The first integral can be simplified (by using the method of integration by parts) to

$$
I_{1}=\frac{1}{2} \int_{-\sigma-\epsilon}^{\sigma+\epsilon} j(m-n) X \exp (j \omega(m-n) X) \mathrm{d}\left(|G(\omega)|^{2}\right) .
$$


12 On the uncertainty inequality as applied to discrete signals

Further simplification yields

$$
\begin{aligned}
I_{1}=\frac{1}{2} j(m-n) X[ & \left.|G(\omega)|^{2} \exp (j \omega(m-n) X)\right|_{-\sigma-\epsilon} ^{\sigma+\epsilon} \\
& \left.-\int_{-\sigma-\epsilon}^{\sigma+\epsilon} j(m-n) X|G(\omega)|^{2} \exp (j \omega(m-n) X) \mathrm{d} \omega\right] .
\end{aligned}
$$

Since $G(-\sigma-\epsilon)$ and $G(\sigma+\epsilon)$ are zero, the first term of (3.18) vanishes. The second term of (3.18) can be further reduced to obtain

$$
I_{1}=\frac{1}{2}(m-n)^{2} X^{2} \int_{-\sigma-\epsilon}^{\sigma+\epsilon}|G(\omega)|^{2} \exp (j \omega(m-n) X) \mathrm{d} \omega .
$$

Combining $I_{1}$ and $I_{2}$, we get

$$
\begin{aligned}
I_{1}+I_{2} & =\int_{-\sigma-\epsilon}^{\sigma+\epsilon}\left(\frac{1}{2}(m-n)^{2}+m n\right) X^{2}|G(\omega)|^{2} \exp (j \omega(m-n) X) \mathrm{d} \omega \\
& =\frac{\left(m^{2}+n^{2}\right)}{2} X^{2} \int_{-\sigma-\epsilon}^{\sigma+\epsilon}|G(\omega)|^{2} \exp (j \omega(m-n) X) \mathrm{d} \omega .
\end{aligned}
$$

The integral of (3.20) and $I_{3}$ (both together constituting (3.15)) involve the evaluation of integrals of the forms

$$
\begin{aligned}
& A=\int_{-\sigma-\epsilon}^{\sigma+\epsilon}|G(\omega)|^{2} \exp (j \omega(m-n) X) \mathrm{d} \omega, \\
& B=\int_{-\sigma-\epsilon}^{\sigma+\epsilon}\left|\frac{\mathrm{d} G(\omega)}{\mathrm{d} \omega}\right|^{2} \exp (j \omega(m-n) X) \mathrm{d} \omega .
\end{aligned}
$$

The left-hand side of (3.15) can be rewritten as

$$
\int_{-\infty}^{\infty} x g(x-m X) x g(x-n X) \mathrm{d} x=\frac{1}{2 \pi}\left(\frac{m^{2}+n^{2}}{2} X^{2} A+B\right)
$$

where $A$ and $B$ are as defined in (3.21).

From (3.11), the derivative of $G(\omega)$ is given by

$$
\frac{\mathrm{d} G(\omega)}{\mathrm{d} \omega}= \begin{cases}\frac{-X \pi}{2 \epsilon} \sin \left(\frac{\pi(\omega+\sigma)}{\epsilon}\right), & -\sigma-\epsilon \leq \omega<-\sigma, \\ \frac{-X \pi}{2 \epsilon} \sin \left(\frac{\pi(\omega-\sigma)}{\epsilon}\right), & \sigma<\omega \leq \sigma+\epsilon, \\ 0, & \text { elsewhere. }\end{cases}
$$


The expression for $A$ is given by

$$
\begin{aligned}
A= & 2 \sigma X^{2} \delta_{m n} \\
& +\frac{X^{2}(-1)^{(m-n)}}{2}\left(\epsilon \frac{\sin ((m-n) X \epsilon)}{(m-n) X \epsilon}+\frac{\sin ((m-n) X \epsilon)}{2}\left[\frac{2(m-n) X}{(\pi / \epsilon)^{2}-(m-n)^{2} X^{2}}\right]\right) \\
& +\frac{X^{2}(-1)^{(m-n)}}{2}\left(\epsilon \frac{\sin ((m-n) X \epsilon)}{(m-n) X \epsilon}\left\{1-\frac{1 / 2}{\left[1-((m-n) X \epsilon / 2 \pi)^{2}\right]}\right\}\right) .
\end{aligned}
$$

The expression for $B$ is given by

$$
B=\frac{1}{4 \epsilon} \frac{\sin ((m-n) X \epsilon)}{(m-n) X \epsilon} \frac{X^{2} \pi^{2}(-1)^{(m-n)}}{\left[1-((m-n) X \epsilon / 2 \pi)^{2}\right]} .
$$

The effective spatial width of $f(x)$ in terms of its samples is then given by

$$
\left(\Delta_{x}\right)^{2}=\frac{1}{2 \pi} \sum_{m \in \mathbb{Z}} \sum_{n \in \mathbb{Z}} f(m X) f(n X)\left(\frac{m^{2}+n^{2}}{2} X^{2} A+B\right),
$$

where $A$ and $B$ are to be obtained from (3.24) and (3.25), respectively. The square of the space-bandwidth product is then the product of the right-hand sides of (3.26) and (3.8) which in combination with (1.5) give the following version of the Heisenberg inequality of a bandlimited signal explicitly in terms of its samples:

$$
\begin{aligned}
\left(\Delta_{\omega}\right)^{2}\left(\Delta_{x}\right)^{2}= & \frac{1}{4 \pi^{2} X}\left[\frac{2 \pi^{3}}{3} \sum_{n \in \mathbb{Z}} f^{2}[n]+\sum_{\substack { m \in \mathbb{Z} \\
\begin{subarray}{c}{n \in \mathbb{Z} \\
m \neq n{ m \in \mathbb { Z } \\
\begin{subarray} { c } { n \in \mathbb { Z } \\
m \neq n } }\end{subarray}} \frac{4 \pi(-1)^{(m-n)}}{(m-n)^{2}} f[m] f[n]\right] \\
& \times\left[\sum_{m \in \mathbb{Z}} \sum_{n \in \mathbb{Z}} f[m] f[n]\left(\frac{m^{2}+n^{2}}{2} X^{2} A+B\right)\right] \geq \frac{1}{4} .
\end{aligned}
$$

3.2. General observations. We have dealt exclusively with the problem of expressing the standard uncertainty inequality for a continuous-time bandlimited (BL) signal in terms of its correctly sampled version. As explained in the introduction, the motivation for this work arose from some apparent paradoxes in the literature on the uncertainty inequality for discrete sequences as applied to sampled BL signals. Moreover, most of the new definitions of discrete uncertainty (in the literature) seem to be ad hoc.

For BL signals whose correct samples are given, there is one-to-one relationship between the discrete-time signal and the continuous version. That is, the standard uncertainty inequality (1.5), see page 2 , can be recast in terms of the correct samples of the signal in the form (3.27) which is obtained by combining (3.8) with (3.26).

Interestingly, the discrete uncertainty inequality for BL signals has the following structure:

$$
\left(\Delta_{\omega}\right)^{2}\left(\Delta_{x}\right)^{2} \triangleq\left(\underline{f^{\prime} \mathscr{A} f}\right)\left(\underline{f^{\prime} \mathscr{B} f}\right) \geq \frac{1}{4}
$$


where $f$ is the infinite-dimensional vector of samples; prime denotes transpose; and $\mathscr{A}$, $\mathscr{B}$ are infinite-dimensional matrices. The diagonal elements of $\mathscr{A}$ are constant, and the off-diagonal elements are proportional to $(-1)^{(m-n)} /(m-n)^{2}$ for $m \neq n$. On the other hand, the diagonal elements of $\mathscr{B}$ contain a constant term and a term proportional to $m^{2}$; and the off-diagonal elements contain a term proportional to $(\operatorname{sinc}(m-n) X \epsilon)$ along with another proportional to $\left(m^{2}+n^{2}\right)$ for $m \neq n$. The contribution to the off-diagonal elements of $\mathscr{B}$ from $A$ and $B$, see (3.24) and (3.25), depends on $\epsilon$, and it tends to 0 as the number of terms tends to $\infty$.

In this context, if we examine the definitions, in the literature, of discrete uncertainty as applied to general sequences, it is found that none of them possess the structure corresponding to matrices $\mathscr{A}$ and $\mathscr{B}$. This is perhaps one of the reasons for their not reducing to the standard uncertainty inequality as applied to sampled BL signals.

The above interpretation prompts casting of the uncertainty problem for general discrete sequences $f[n]$ for $n=0,1, \ldots, N-1$ as follows.

Problem of uncertainty in discrete signals. Assuming that

$$
\sum_{n=0}^{N-1}|f[n]|^{2}=1,
$$

find the lower bound (if any) on

$$
\left(\underline{f^{\prime} \mathscr{A} f}\right)\left(\underline{f^{\prime} \mathscr{B} f}\right)
$$

where the $N$-by- $N$ matrices $\mathscr{A}$ and $\mathscr{B}$ have the following structure.

(1) The diagonal elements of $\mathscr{A}$ are 1 , and the off-diagonal elements are proportional to $(-1)^{(m-n)} /(m-n)^{2}$ for $m \neq n$.

(2) The diagonal elements of $\mathscr{B}$ contain a constant term and a term proportional to $m^{2}$; and the off-diagonal elements contain a term proportional to $(\operatorname{sinc}(m-$ $n) X \epsilon$ ) (where $\epsilon$ is a parameter) along with another proportional to $\left(m^{2}+n^{2}\right)$ for $m \neq n$. The parameter $\epsilon$ facilitates generalization of the result to the samples of continuous-time bandlimited signals.

Note that for perfect reconstruction of the bandlimited signal, $\epsilon$ can be any arbitrary value in the interval $(0, \pi / X-\sigma)$ (see the last sentence of Section 3.1, item (1)). In this structure, the off-diagonal elements of $\mathscr{B}$ are to be the limit values (which in this case would be zero) obtained from allowing $\epsilon \rightarrow 0$. In addition, a necessary condition for finite spatial width is that $F( \pm \sigma)=0$. Note that the discrete signal having finite samples still corresponds to a BL signal. If the above, newly defined, discrete uncertainty is to be applicabe to the samples of a general bandlimited signal, then $N \rightarrow \infty$. A comparison of the result so obtained with the contents of the paper of Donoho and Huo [8] would be quite enlightening.

It is also interesting to explore a possible relationship between the discrete uncertainty (for samples of BL signals) and affine uncertainty [2] that has been defined for general square integrable functions which cannot be sampled unless they are bandlimited. In the latter case, it should be possible to relate the continuous affine uncertainty conditions (of the type found in [2]) to those of discrete uncertainty. 
The choice of the (interpolating function) $G$ does not affect the lower uncertainty bound. The $G$ actually chosen in the paper is merely one out of many interpolating functions that ensure perfect reconstruction, while simultaneously having appropriate smoothness properties. For analytical tractability, some forms of $G$ seem to be more advantageous than the others, that is, the continuous version of the uncertainty inequality can be easily expressed in terms of the (correct) samples of the BL function. The discrete spreads computed perfectly match with the corresponding continuous ones irrespective of the choice of $G$. Note that the standard sinc-function cannot be used in this operation because its effective time- (or space-) spread is $\infty$ which is reflected in the nondifferentiability of its transform.

Further, we do not (in this paper) examine the choice of an optimal $G$ which can theoretically be determined by solving the following problem.

Optimal interpolating function. Find a $G$ function which obeys the smoothness and bandlimitedness conditions on pages $9-10$ such that the following product is minimized:

$$
\left(\int_{-\infty}^{\infty} x^{2} g^{2}(x) d x\right)\left(\int_{-\infty}^{\infty} \omega^{2} G^{2}(\omega) d x\right)
$$

subject to the constraint that $G$ is also an interpolating function.

A solution to this problem would give the $G$ function with optimal localization properties. However, the discrete version of the uncertainty inequality is still (3.27). There seems to be no need for invoking frame theory currently being used in the literature on wavelet transforms.

A byproduct of our approach (to discrete signal uncertainty as applied to BL signals) is that we can analyze the problem of lower uncertainty bound and the design of discretetime signals with minimum space-bandwidth product. This work will appear in another paper. On the other hand, as explained above, we can formulate the problem of discrete uncertainty in such a way that when applied to the sampled BL signals, the computed value of the square of the space-bandwidth product, that is, the left-hand side of inequality (3.27), is the same as the left-hand side of (1.5) of the continuous version. Since $G$ is bandlimited, we can express its effective (spatial and spectral) spreads using its discrete samples. Moreover, $G$ is an interpolating function for reconstruction of the signal from its discrete samples; and, as a consequence, $G$ is not merely an analyzing atom like the ones encountered in wavelet or Gabor representation of signals.

From yet another point of view, we can try to find the discrete signal by minimizing uncertainty defined in an appropriate manner (different from what has been presented above). We have also obtained some interesting results in this regard which will be published separately.

In the next section, we compare the results obtained from an application of (3.27) with those of the literature.

\section{Computational results}

We now present a few typical results of computation for synthetic, bandlimited signals. The numbers computed are the SBPs obtained (a) theoretically from the original 
16 On the uncertainty inequality as applied to discrete signals

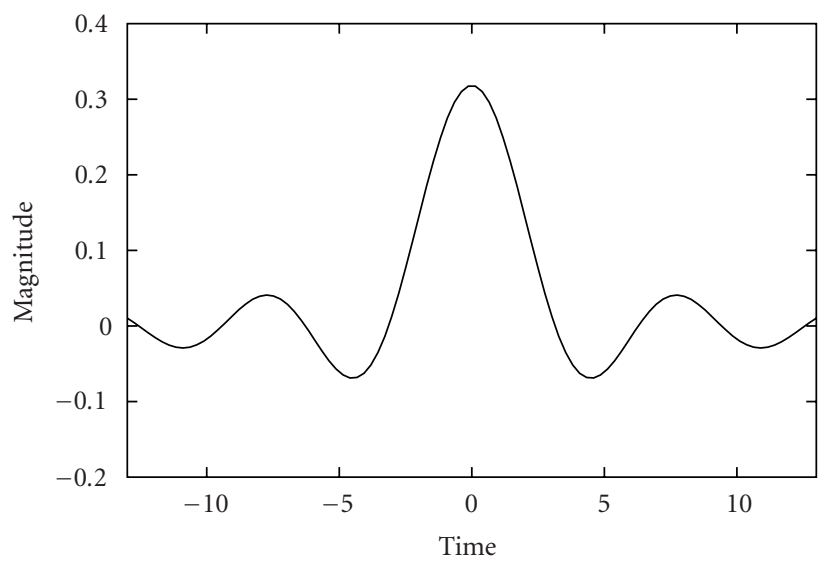

Figure 4.1. sinc-function.

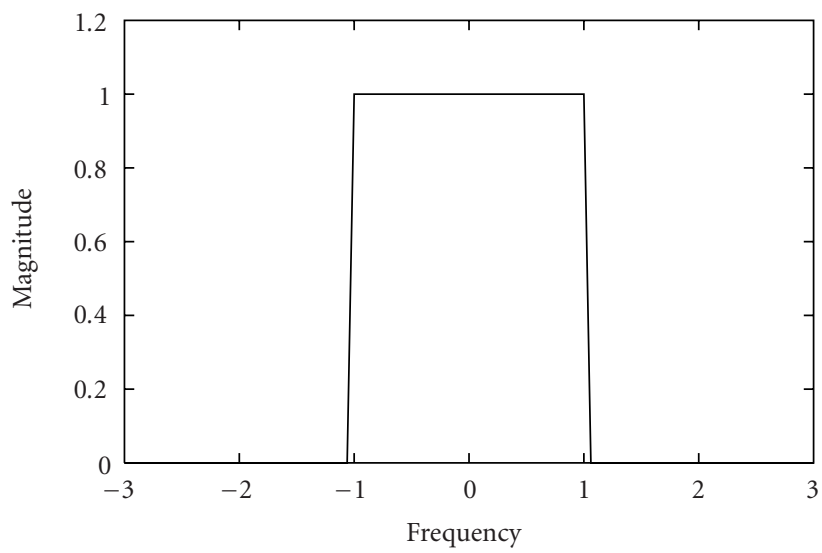

Figure 4.2. Spectrum of sinc-function.

continuous-space function; and, from the samples, by using (b) Doroslovački's definition; and (c) our definitions.

Figures 4.1, 4.4, and 4.7 show some typical bandlimited continuous signals. The continuous Fourier spectrum of the above-mentioned signals is shown in Figures 4.2, 4.5, and 4.8, respectively. These signals are sampled correctly based on the Shannon theorem. The sampled versions are shown in Figures 4.3, 4.6, and 4.9. The signals have theoretical effective spatial and spectral widths (as computed by using (1.2) and (1.3)) given in columns 2 and 5, respectively, of Table 2.1. In the same table, we give, the effective widths as obtained from samples using our results, along with those obtained from Doroslovački's definition for the discrete signal. Table 4.1 contains the SBP as obtained directly from (1.2) and (1.3) along with the SBP from the samples using our results. 


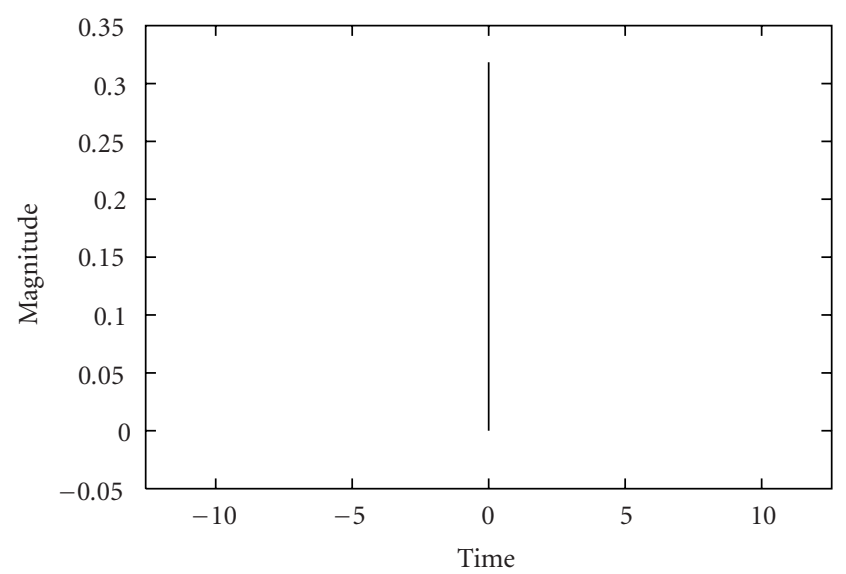

Figure 4.3. Samples of sinc-function.

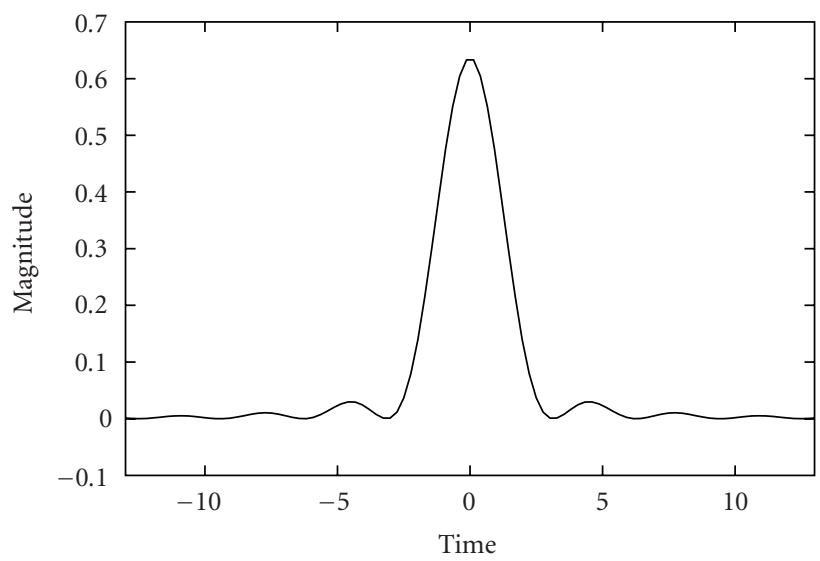

Figure 4.4. sinc-square function.

It is to be observed here that there is a discrepancy between the correct value of the effective spatial and spectral widths (directly computed from (1.2) and (1.3) for the original, bandlimited continuous signal) and the results of Doroslovački for the sampled version of the same signal. In contrast with this, the proposed technique gives exactly (except for numerical errors in digitization) the same effective spreads, and hence the same uncertainty product when the samples of the signals are used. Note that the products are greater than the lower limit $1 / 4$ for normalized signals.

\section{Optimal bandlimited signals}

Here we present some experimental findings related to the second problem posed in the introduction. Let $\mathscr{B}$ denote the set of all finite-(unit-) energy bandlimited functions. We 
18 On the uncertainty inequality as applied to discrete signals

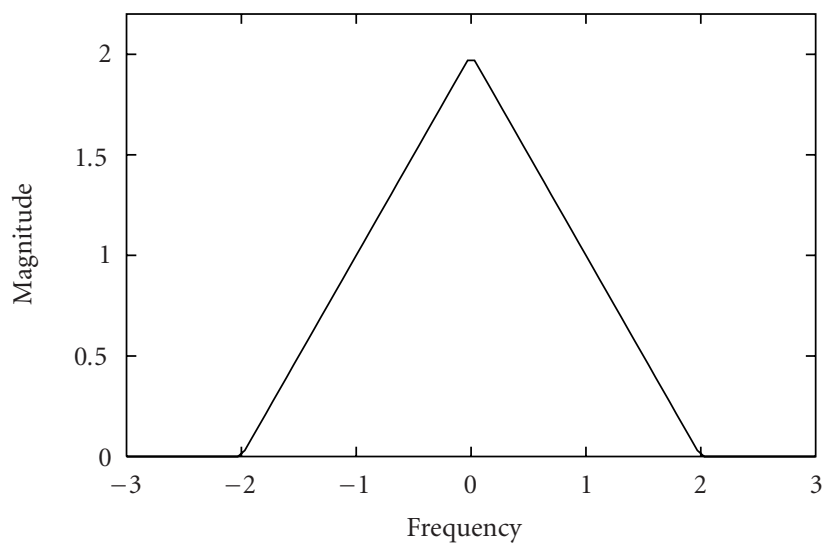

Figure 4.5. Spectrum of sinc-square function.

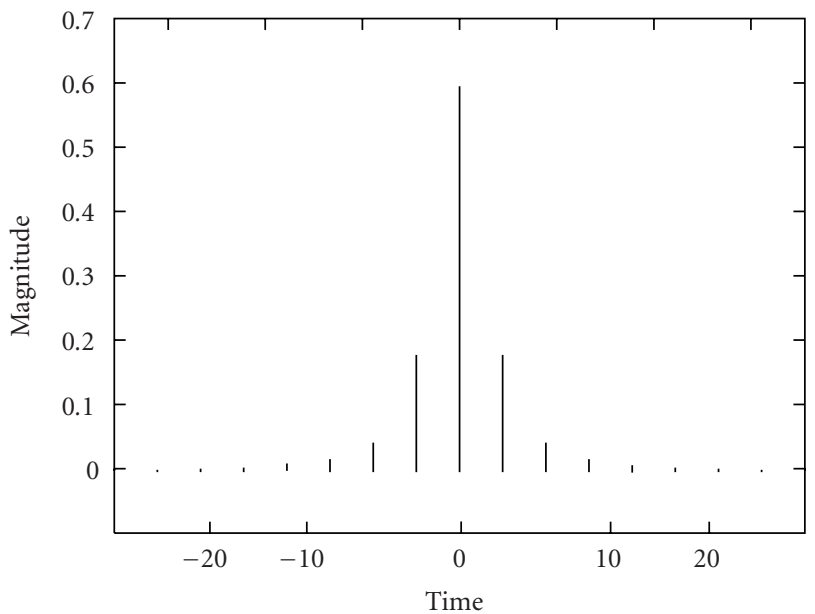

Figure 4.6. Samples of sinc-square function.

Table 4.1. Uncertainty product for various signals of unit energy.

\begin{tabular}{|c|c|c|c|c|}
\hline & & Continuous & Discrete & \\
\hline & & Gabor's Def. & Doroslovački's Def. & Proposed Def. \\
\hline no. & Function & Uncertainty product & Uncertainty product & Uncertainty product \\
\hline 1 & $\sin c$ & $\infty$ & Not applicable & $\infty$ \\
\hline 2 & Triangle & 0.29730 & 0.06501 & 0.30278 \\
\hline 3 & Halfcos & 0.32245 & 0.00403 & 0.32302 \\
\hline
\end{tabular}

provide an answer to a slightly modified question: how close can the uncertainty product of functions belonging to $\mathscr{B}$ get to the lower bound obtained in (1.5)? 


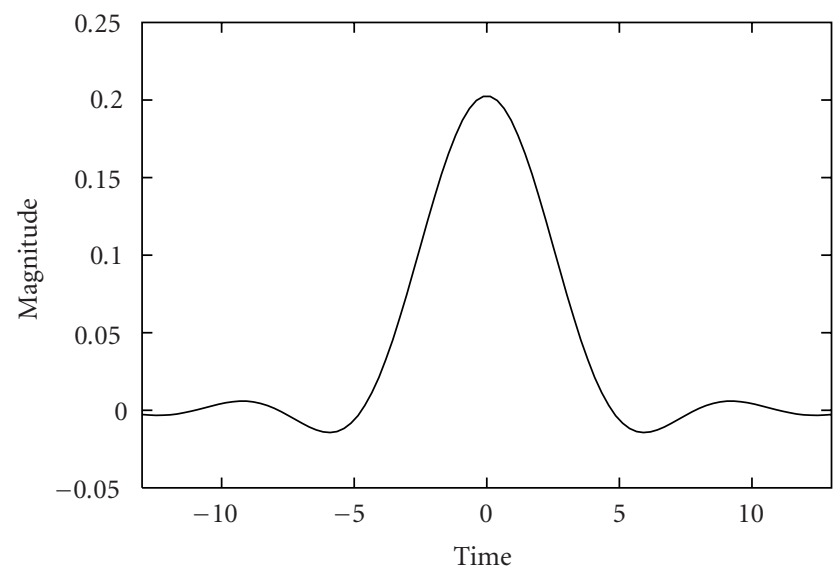

Figure 4.7. Halfcos continuous function.

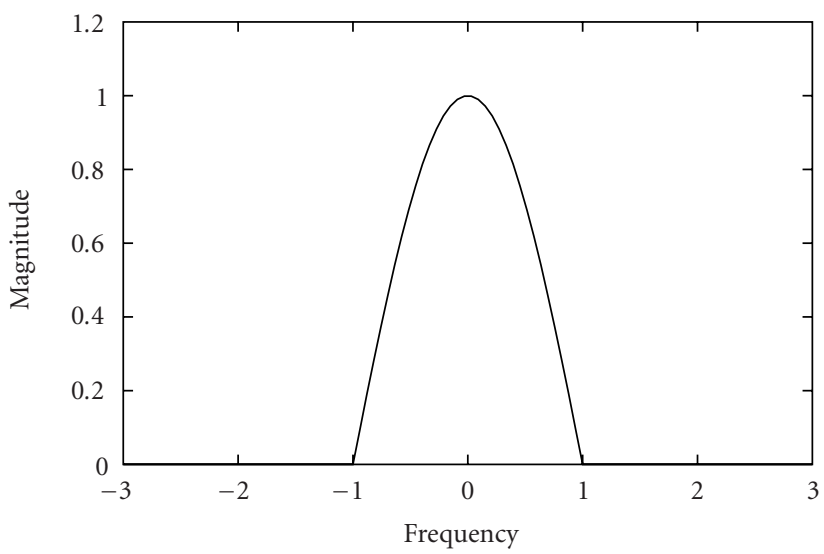

Figure 4.8. Halfcos spectrum.

Using (1.2) and the properties of Fourier transform, it can be shown that the effective spatial width is finite only if $|d F(\omega) / d \omega|$ is square integrable. Therefore, for $f \in \mathscr{B}$, we require that $F(\omega)$ have finite derivatives at $\omega=W$ and $\omega=-W$ (i.e., at bandlimit points). We now provide a solution (to the above problem) based on a modification of the Gaussian function. Let

$$
F(\omega)=\Phi(\omega) G(\omega)
$$

where

$$
\Phi(\omega)=C \exp \left(\frac{-\omega^{2}}{2 \sigma^{2}}\right)
$$

where $C$ is a positive constant, $\sigma$ is the variance parameter, and $G(\omega)$ is defined in $(3.11)$. 


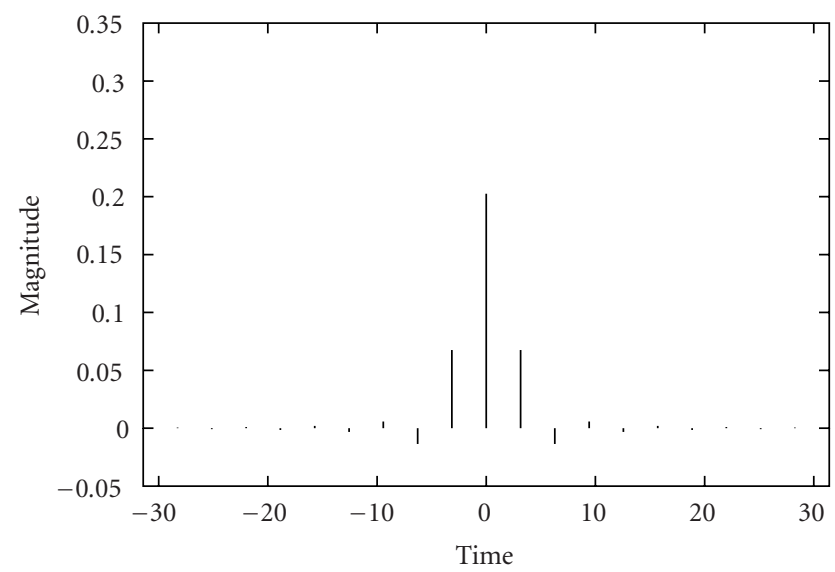

Figure 4.9. Halfcos discrete function.

Table 5.1. Convergence of uncertainty product using cosine interpolation function with $\epsilon=0.1$ and $W=\pi$.

\begin{tabular}{lcc}
\hline no. & $\sigma$ & Product \\
\hline 1 & 2.0 & 1.19870 \\
2 & 1.8 & 0.74649 \\
3 & 1.7 & 0.57802 \\
4 & 1.5 & 0.36008 \\
5 & 1.3 & 0.27172 \\
6 & 1.0 & 0.25026 \\
7 & 0.9 & 0.25002 \\
8 & 0.8 & 0.25000 \\
9 & 0.7 & 0.25000 \\
10 & 0.5 & 0.25000 \\
\hline
\end{tabular}

With this choice of the functions on the right-hand side of (5.1), $F(\omega)$ is a bandlimited function, with (a) the bandlimit $W+\epsilon$; (b) $F(\omega)=0$, for all $|\omega|=W+\epsilon$; and (c) $F(\omega)$ differentiable everywhere. Tables 5.1 and 5.2 give the numerical values of SBP of $f$ for various values of $\sigma$. Note that there is a sequence of bandlimited functions whose SBP approaches the lower bound in (1.5) for a given bandwidth $W$ and $\epsilon$. It should be emphasized that these results are purely computational, and an analytical solution to the problem under consideration seems to be unknown.

Remarks. (1) The equality in (1.5) is attained only by the Gaussian function, which is not bandlimited. Therefore, any bandlimited function has an SBP strictly greater than $1 / 4$.

(2) It is interesting to note that for any small value of $\epsilon>0$, there are bandlimited functions (defined by (5.1)) whose SBP is very close to $1 / 4$. Further, the SBP could as well be $1 / 4$, this phenomenon having been possibly caused by the finite precision of the computer. 
Table 5.2. Convergence of uncertainty product using cosine interpolation function with $\epsilon=0.01$ and $W=\pi$

\begin{tabular}{lcr}
\hline no. & $\sigma$ & Product \\
\hline 1 & 2.0 & 10.42400 \\
2 & 1.8 & 5.66300 \\
3 & 1.7 & 3.86440 \\
4 & 1.5 & 1.49740 \\
5 & 1.3 & 0.50648 \\
6 & 1.0 & 0.25349 \\
7 & 0.9 & 0.25031 \\
8 & 0.8 & 0.25001 \\
9 & 0.7 & 0.25000 \\
10 & 0.5 & 0.25000 \\
\hline
\end{tabular}

\section{Conclusions}

We have described some of the existing definitions for the spatial and spectral spreads for discrete signals, and showed that for bandlimited signals, they do not correspond to the standard definitions of spatial and spectral widths in the continuous domain. A new approach has been proposed to overcome this limitation and to express the original Heisenberg inequality in terms of the samples of the bandlimited signal.

An unsolved problem is the determination of a bandlimited signal for which the SBP (obtained by multiplying the effective widths defined by (1.2) and (1.3)) is minimal. A possible approach to solve this problem is the representation of a general bandlimited signal as linear combination of prolate spheroidal functions as a basis [15, pages 205215].

\section{Acknowledgment}

The authors wish to express their grateful thanks to the reviewers and to the Editor-inChief for their valuable remarks, suggestions, and questions which have led to the present improved version.

\section{References}

[1] L. C. Calvez and P. Vilbe, On the uncertainty principle in discrete signals, IEEE Transactions on Circuits and SystemsPart II: Analog and Digital Signal Processing 6 (1992), no. 39, 394-395.

[2] S. Dahlke and P. Maass, The affine uncertainty principle in one and two dimensions. Concrete analysis, Computers \& Mathematics with Applications 30 (1995), no. 3-6, 293-305.

[3] L. Debnath (ed.), Wavelet Transforms and Time-Frequency Signal Analysis, Applied and Numerical Harmonic Analysis, Birkhäuser Boston, Massachusetts, 2001.

[4] L. Debnath, Wavelet Transforms and Their Applications, Birkhäuser Boston, Massachusetts, 2002.

[5] L. Debnath (ed.), Wavelets and Signal Processing, Applied and Numerical Harmonic Analysis, Birkhäuser Boston, Massachusetts, 2003.

[6] V. DeBrunner, M. Özaydin, and T. Przebinda, Resolution in time-frequency, IEEE Transactions on Signal Processing 47 (1999), no. 3, 783-788. 
[7] A. Dembo, T. M. Cover, and J. A. Thomas, Information-theoretic inequalities, IEEE Transactions on Information Theory 37 (1991), no. 6, 1501-1518.

[8] D. L. Donoho and X. Huo, Uncertainty principles and ideal atomic decomposition, IEEE Transactions on Information Theory 47 (2001), no. 7, 2845-2862.

[9] D. L. Donoho and P. B. Stark, Uncertainty principles and signal recovery, SIAM Journal on Applied Mathematics 49 (1989), no. 3, 906-931.

[10] M. I. Doroslovački, Product of second moments in time and frequency for discrete-time signals and the uncertainty limit, Signal Processing 67 (1998), no. 1, 59-76.

[11] M. I. Doroslovački, H. Fan, and P. M. Djuric, Time-frequency localization for sequences, Proceedings of the IEEE-SP International Symposium on Time-Frequency and Time-Scale Analysis, Victoria, British Columbia, 1992, pp. 159-162.

[12] G. B. Folland and A. Sitaram, The uncertainty principle: a mathematical survey, The Journal of Fourier Analysis and Applications 3 (1997), no. 3, 207-238.

[13] F. J. Harris, On the use of windows for harmonic analysis with the discrete Fourier transform, Proceedins of IEEE 66 (1978), no. 1, 51-83.

[14] R. Ishii and K. Furukawa, The uncertainty principle in discrete signals, IEEE Transactions on Circuits and Systems 33 (1986), no. 10, 1032-1034.

[15] A. Papoulis, Signal Analysis, McGraw-Hill, New York, 1968.

[16] T. Przebinda, V. DeBrunner, and M. Özaydin, Using a new uncertainty measure to determine optimal bases for signal representations, Proceedings of IEEE International Conference on Acoustics, Speech, and Signal Processing, vol. 3, Arizona, 1999, pp. 1365-1368.

[17] _ The optimal transform for the discrete Hirschman uncertainty principle, IEEE Transactions on Information Theory 47 (2001), no. 5, 2086-2090.

Y. V. Venkatesh: Computer Vision and Artificial Intelligence Laboratory,

Department of Electrical Engineering, Indian Institute of Science, Bangalore 560012, India Current address: Department of Electrical and Computer Engineering, Faculty of Engineering,

National University of Singapore, Singapore 117576

E-mail address: eleyvv@nus.edu.sg

S. Kumar Raja: Computer Vision and Artificial Intelligence Laboratory,

Department of Electrical Engineering, Indian Institute of Science, Bangalore 560012, India

E-mail address: kumar@darbar.ee.iisc.ernet.in

G. Vidyasagar: Computer Vision and Artificial Intelligence Laboratory,

Department of Electrical Engineering, Indian Institute of Science, Bangalore 560012, India

E-mail address: sagar@bhaskara.ee.iisc.ernet.in 


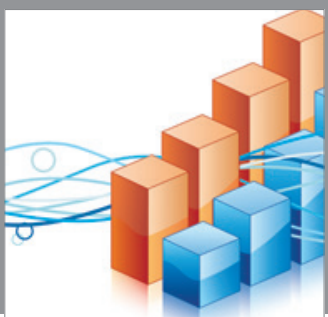

Advances in

Operations Research

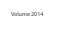

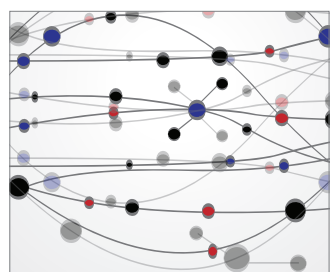

\section{The Scientific} World Journal
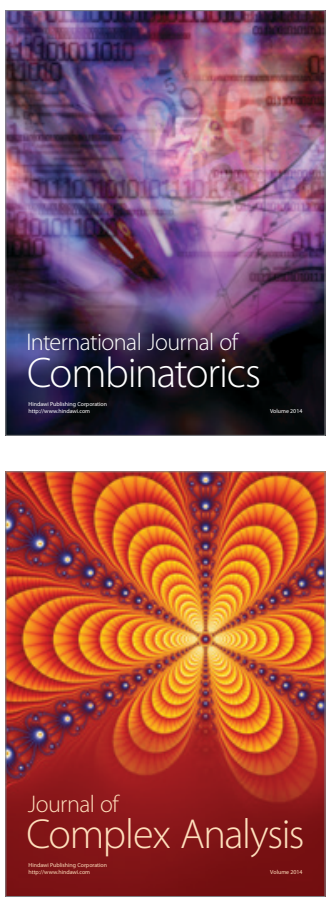

International Journal of

Mathematics and

Mathematical

Sciences
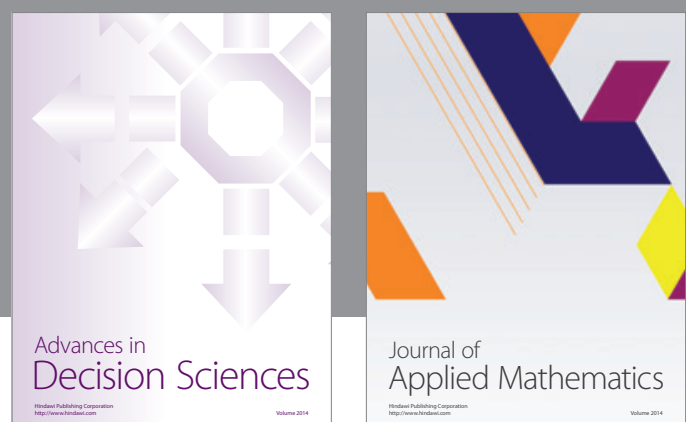

Journal of

Applied Mathematics
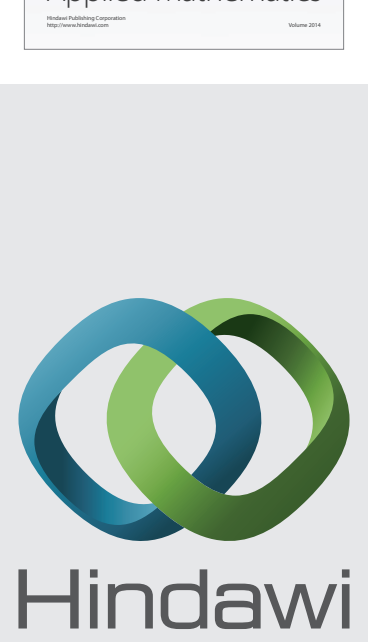

Submit your manuscripts at http://www.hindawi.com
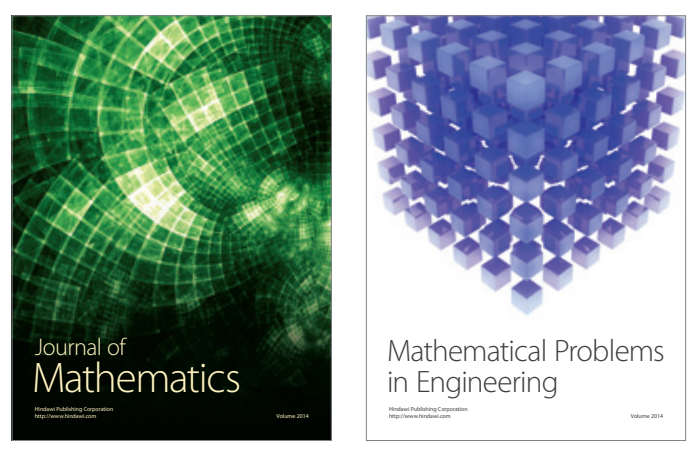

Mathematical Problems in Engineering
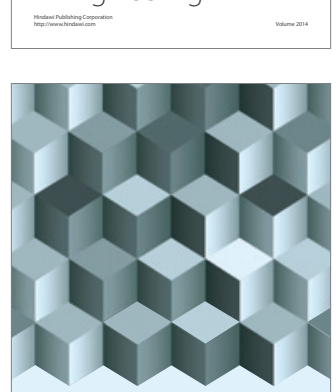

Journal of

Function Spaces
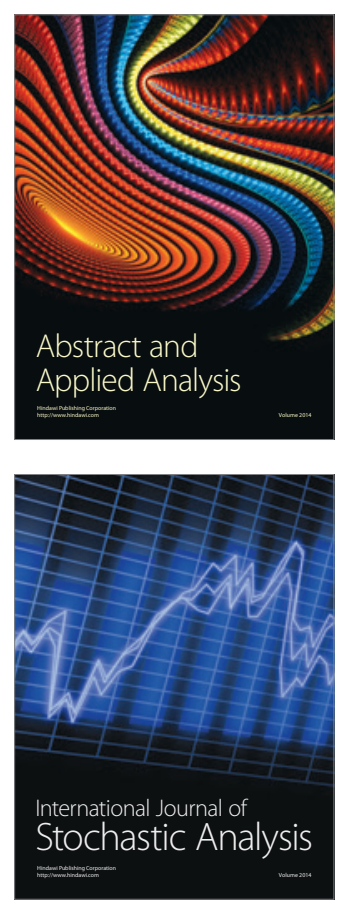

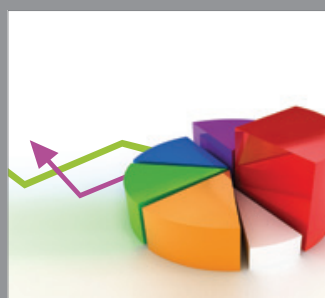

ournal of

Probability and Statistics

Promensencen
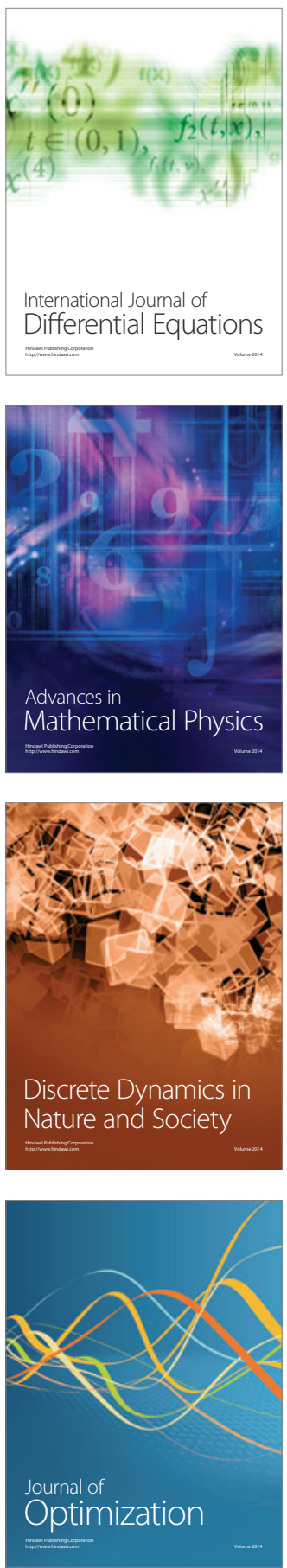